\title{
Explanation of Onset Ferroelectric Transition and Anomalous Tangent Delta in H- Bonded Rubidium Dihydrogen Phosphate Crystal
}

\author{
Kuldeep Kumar $^{1 *}$ Trilok Chandra Upadhyay $^{1}$ \\ ${ }^{1}$ Department of Physics, HNBGU (A Central University) Srinagar UK, India-246174 \\ *Corresponding Author: kuldeep26811@gmail.com
}

Received: 28.8.2021; Revised: 19.9.2021; Accepted: 29.9 2021

(C) Society for Himalayan Action Research and Development

\begin{abstract}
The H-bonded ferroelectric and ordered-disordered, type of Rubidium Dihydrogen Phosphate crystal is theoretically investigated. It undergoes a ferroelectric phase transition at 146K. Modified earlier author's (Ganguli et al, 1980) simple Pseudo Spin Lattice Coupled mode (PLCM) model Hamiltonian by adding some extra interactions term into it, like third-order and fourth-order, phonon anharmonic interactions, direct spin-spin terms, extra spin-lattice terms and four spin coupling terms are added. By using Zuberav's statistical approach, two times temperature dependent Green function method, and Dyson's equation in the mean-field approximation (MFA), to obtain the formulae of various quantities like $(\varepsilon)$, $\Omega \operatorname{and} \tan (\delta)$, Curie's temperature, and response function (shift and width) as a function of temperature. Thermal variations of above quantities are obtained for Rubidium Dihydrogen Phosphate $\left(\mathrm{RbH}_{2} \mathrm{PO}_{4}\right)$ crystal. A comparison with theoretical findings has been made with the experimental findings reported by (Peercy et al, 1974) which shows good agreement.
\end{abstract}

Keywords: Electrical permittivity, Ferroelectricity, Curie's temperature, Green function, and Response function.

\section{Introduction}

The rubidium dihydrogen phosphate crystal is abbreviated as RDP. In the investigations of ferroelectric $\mathrm{RbH}_{2} \mathrm{PO}_{4}$ crystal of great interest aroused in the past few years as they have got promising applications in a wide range of areas, due to their interesting properties which are widely used in laser technologies, memory devices, etc. The alkali phosphate H-bonded ferroelectric crystals are very famous called $\mathrm{KH}_{2} \mathrm{PO}_{4}$ type crystals because of their isomorphic structure, and their deuterated structure is also fully or partially analogous.

The $\mathrm{RbH}_{2} \mathrm{PO}_{4}$ crystal is characterised by covalently bonded $\mathrm{PO}_{4}$ units connected by bridging H-bonds and ionic bonding between rubidium cations and $\mathrm{PO}_{4}$ anions, which is responsible for the formation of the minima of double-well potential. The $\mathrm{RbH}_{2} \mathrm{PO}_{4}$ crystal is undergone phase transition at $\mathrm{T}_{\mathrm{c}}=146 \mathrm{~K}$. In the paraelectric phase when $\left(T>T_{c}\right)$ then Oxygen atoms of the Hydrogen bond are located by an axis $\mathrm{C}_{2}$ which implies that the Hydrogen atoms are disordered between two symmetrical positions along with Oxygen-Hydrogen-Oxygen bonds with equal probability. In the ferroelectric phase when $\left(, T<T_{c}\right)$ all of the protons are ordered into one of the double-well potentials. The lattice parameters of $\mathrm{RbH}_{2} \mathrm{PO}_{4}$

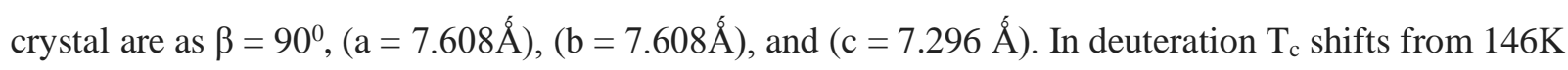
to $249.8 \mathrm{~K}$ which shows a large isotopic effect. It is an orthorhombic structure with the $\mathrm{PO}_{4}$ groups are connected by $\mathrm{O}-\mathrm{H}---\mathrm{O}$ bonds below $\mathrm{T}_{\mathrm{c}}$, and a tetragonal structure with the four $\mathrm{Rb}^{-} \mathrm{PO}_{4}$ groups in the 
unit cell above $\mathrm{T}_{\mathrm{c}}$. The (spontaneous polarization) in the $\mathrm{RbH}_{2} \mathrm{PO}_{4}$ crystal is perpendicular to the direction of the Oxygen-Hydrogen-Oxygen bond and $\mathrm{PO}_{4}$ group in $\mathrm{RbH}_{2} \mathrm{PO}_{4}$ crystal, bonding in three dimensional. (Cochran, 1960) has given the theory of transition in terms of instability against one of the normal modes of vibrations. The experimental and theoretical studies on $\mathrm{RbH}_{2} \mathrm{PO}_{4}$ type crystals have been made by many workers. (Blinc, 1960) first explained the isotope effect in $\mathrm{RbH}_{2} \mathrm{PO}_{4}$ crystal. (Averbuch and Durif, 1985) have studied the new structure form of $\mathrm{RbH}_{2} \mathrm{PO}_{4}$ crystals. (Peercy, 1974) has studied coupled proton-optic mode model for $\mathrm{RbH}_{2} \mathrm{PO}_{4}$ and $\mathrm{KH}_{2} \mathrm{PO}_{4}$ crystals and calculated the spontaneous polarization, Curie's temperature, and electrical permittivity. In $\mathrm{RbH}_{2} \mathrm{PO}_{4}$ crystal, a Raman study has been done by (Courtens and Vogt, 1985). (Adhav, 1969) has studied the phase transition, piezoelectric and elastic properties in $\mathrm{KH}_{2} \mathrm{PO}_{4}$ type H-bonded crystal. (Semwal and Sharma, 1974) have studied the infrared absorption in H- bonded anharmonic crystal and susceptibility by using (PLCM) model and Green function technique as a function of two-time temperature. (Agui et al, 1993) have studied the hydrogen bonds and phosphate $\left(\mathrm{PO}_{4}\right)$ mode in $\mathrm{KH}_{2} \mathrm{PO}_{4}$ and $\mathrm{RbH}_{2} \mathrm{PO}_{4}$ crystals which have been investigated by using the inelastic neutron scattering and Raman scattering experiments. (Dulterio and Oweni, 1988) have studied phase transition at high temperature in $\mathrm{RbH}_{2} \mathrm{PO}_{4}$ crystals. (Upadhyay and Semwal, 2002) have studied dielectric properties in H-bonded crystals. (Mattauch et al, 2011) have studied $\mathrm{x}$-ray diffraction and high-resolution neutron as a function of temperature in $\mathrm{KH}_{2} \mathrm{PO}_{4}$ type $\mathrm{H}$ bonded crystals. (Boysen et al, 2004) have studied conductivity at high temperature and pressure. Dielectric properties in $\mathrm{H}$ - bonded crystal of $\mathrm{KH}_{2} \mathrm{PO}_{4}$ family studied by (Singh et al, 2021 and Kahan and Upadhayay, 2020). (Ganguli et al, 1980) have studied the phase transitions in $\mathrm{KH}_{2} \mathrm{PO}_{4}$ type $\mathrm{H}$ bonded crystals by using the (PLCM) model and Green's function technique. They modified the earlier author's model (Zuberav's, 1960) by adding extra terms and explained the permittivity of $\mathrm{KH}_{2} \mathrm{PO}_{4}$ type crystals. (Singh et al, 2021) have not considered the four spin coupling term in their model to explain the dielectric properties of $\mathrm{RbH}_{2} \mathrm{PO}_{4}$ crystals. Earlier authors (Singh et al, 2021 and Kahan and Upadhayay, 2020) have not considered the four spin coupling term in their model to explain the ferroelectric properties of the $\mathrm{RbH}_{2} \mathrm{PO}_{4}$. In contrast to their treatment (decoupling), we shall use a better decoupling scheme, which will enhance the terms in the results.

In this work, we considered the extra terms like direct spin-spin interaction term, extra spin-phonon interaction term and (third-order and fourth-order lattice anharmonic interactions terms), in the (PLCM) model for $\mathrm{RbH}_{2} \mathrm{PO}_{4}$ crystals to find out theoretical expressions. These extra terms play an important role in explaining the thermal dependent quantities of Cochran's mode frequency, electrical permittivity, tangent delta, shift-width and tangent delta. We have compared our theoretical calculated findings with the experimental finding of (Peercy et al, 1974). 


\section{Modified Hamiltonian and Calculation}

For the investigation of ferroelectric properties in $\mathrm{RbH}_{2} \mathrm{PO}_{4}$ crystals, we consider the modified model as;

$$
H_{1}=-2 \Omega \sum_{i} S_{i}^{x}-\frac{1}{2} \sum_{i j} J_{i j} S_{i}^{z} S_{j}^{z}+\frac{1}{4} \sum_{k} \omega k\left(A_{k}^{\dagger} \mathrm{A}_{\mathrm{k}}+\mathrm{B}_{\mathrm{k}}^{\dagger} \mathrm{B}_{\mathrm{k}}\right)-\sum_{\mathrm{ik}} \mathrm{V}_{\mathrm{ik}} \mathrm{S}_{\mathrm{i}}^{\mathrm{Z}} \mathrm{A}_{\mathrm{k}},
$$

And

$$
\begin{aligned}
& H_{2}=\sum_{k_{1} k_{2} k_{3}} V^{3}\left(k_{1}, k_{2}, k_{2}\right) A_{k_{1}} A_{k_{2}} A_{k_{3}}+\sum_{k_{1} k_{2} k_{3} k_{4}} V^{4}\left(k_{1}, k_{2}, k_{3}, k_{4}\right) A_{k_{1}} A_{k_{2}} A_{k_{3}} A_{k_{4}} \\
& -\frac{1}{2} \sum_{j} B_{i j} S_{i}^{x} S_{j}^{x}+\frac{1}{4} \sum_{i j k l} J_{i j k l}^{\prime} S_{i}^{z} S_{j}^{z} S_{k}^{z} S_{l}^{z}-\sum_{i k} V_{i k} S_{i}^{x} A_{k}-\sum_{i k} V_{i k} S_{i}^{x} A_{k}^{2},
\end{aligned}
$$

where, $\Omega$ represents the proton tunnelling frequency, $J_{i j}$ is the interaction constant, $V_{\mathrm{ik}}$ represent spin-lattice interaction constant, $A_{k}$ and $B_{k}$ are the displacement operator and the momentum operator, $A_{k}^{\dagger}$ and ${ }_{B}^{\dagger}$ are conjugate of momentum and displacement operators, and $\omega_{k}$ is the phonon harmonic frequency. $V^{3} k_{1}, k_{2}, k_{3}$, and $V^{4} k_{1}, k_{2}, k_{3}, k_{4}$ represents (third-order and fourth-order) lattice's phonon anharmonic interaction terms, $J_{i j k l}^{\prime}$ represent the four body interaction constant, $S_{i}^{x} A k$ and $S_{i}^{x} A_{k}^{2}$ terms represent indirect coupling, and $B_{i j}$ term represents the coupling constant.

We shall add in $\mathrm{H}_{1}$, the $\mathrm{H}_{2}$ given by

$$
\mathrm{H}=\mathrm{H}_{1}+\mathrm{H}_{2} \text {. }
$$

Now by using two-times temperature-dependent statistical method of Green's function technique by (Zubrerav's, 1960) and double differentiation of w.r.t ( $\mathrm{t}^{\prime}$ ) and (t) of Hamiltonian to obtain Dyson's equation as;

$$
\begin{aligned}
& G_{i j} \omega=G_{i j}^{0} \omega+G_{i j}^{0} \omega P \omega G_{i j}^{0} \omega, \\
& { }^{\text {we obtain }} G_{i j}^{0} \omega=\Omega<S_{i}^{x}>\delta_{i j} \pi^{-1}\left(\omega^{2}+\Omega^{2}\right), \\
& P \omega=\pi \Omega^{-1}<S_{i}^{x}{ }^{-1}<<F_{i} t ; F_{j} t^{\prime}>>
\end{aligned}
$$

where $F_{i}(t)$ and $F_{i}\left(t^{\prime}\right)$ are given as

$$
F_{i} t=2 \Omega \sum_{i} V_{i k} A_{k} \sum_{i k} S_{i}^{z}+2 \Omega \sum_{i} V_{i k} A_{k}^{2} \sum_{i k} S_{i}^{z}-\Omega \sum_{i} B_{i j} \sum_{i j} S_{i}^{z} S_{j}^{x}+\delta_{i j} S_{i}^{z} S_{i}^{x}-\ldots
$$


and

$$
F_{i} t^{\prime}=2 \Omega \sum_{i} V_{i k} A_{k} \sum_{i k} S_{i}^{z}+2 \Omega \sum_{i} V_{i k} A_{k}^{2} \sum_{i k} S_{i}^{z}-\Omega \sum_{i} B_{i j} \sum_{i j} S_{i}^{z} S_{j}^{x}+\delta_{i j} S_{i}^{z} S_{i}^{x}-. .
$$

Where $\mathrm{G}^{0}(\omega)$ in Eq. (5) is the zeroth-order Green's function, and are obtained as;

$$
G_{i j}^{0} \omega=\Omega\left\langle S_{i}^{x}\right\rangle \delta_{i j}\left[\pi\left(\omega^{2}-\Omega^{2}\right)^{-1}\right] .
$$

In Eq. (6) $\mathrm{P}(\omega)$ are the response function and $\Omega$ is the normalized pseudo-spin and different product of operators like $\Omega J S_{i j}^{x} S_{j}^{x}, \Omega J{ }_{i j} S_{i}^{z} S_{j}^{x}$ and $A, A^{\dagger}$ etc. contained in Eqn. (7) and (8) which are evaluated after applied decoupling scheme as;

$$
\langle\langle\mathrm{EF} ; \mathrm{GH}\rangle\rangle=\langle E F\rangle\langle\langle\mathrm{G} ; \mathrm{H}\rangle\rangle+\langle E G\rangle\langle\langle\mathrm{F} ; \mathrm{H}\rangle\rangle+\langle E \boldsymbol{H}\rangle\langle\langle\mathrm{F} ; \mathrm{G}\rangle\rangle .
$$

Now, we resolve $P(\omega)$ into its real $\Delta(\omega)$ and imaginary $\Gamma(\omega)$ parts in which the real part is called (shift) and the imaginary part is called (width). We, therefore, obtain the quantities of $\Delta(\omega)$ and $\Gamma(\omega)$ is expressed as $\mathrm{P}(\omega)=\Delta(\omega)+\mathrm{i} \Gamma(\omega)$, and the quantities of delta and gamma are as follows

$$
\Delta \omega=\frac{4 \Omega^{2} V_{i k}^{2} N k}{\left(\omega^{2}-\Omega^{2}\right)}+\frac{4 \Omega a V_{i k}^{2}\left\langle S_{i}^{z}\right\rangle\left(\omega^{2}-\omega_{k}^{2}\right) \omega k}{b\left[\left(\omega^{2}-\widetilde{\omega}_{k}^{2}\right)^{2}-4 \omega_{k}^{2} \Gamma_{k}^{2} \omega\right]}+\frac{B_{i j}^{2} \Omega a J_{0}\left\langle s^{z}\right\rangle^{2}}{b\left(\omega^{2}-\Omega^{2}\right)}+\frac{J_{i j}^{2} \Omega\left\langle s^{z}\right\rangle^{2} a}{2 b\left(\omega^{2}-\Omega^{2}\right)}+. .
$$

Therefore total $\Delta(\omega)=\Delta_{1}(\omega)+\Delta_{2}(\omega)+\ldots$.

Now we calculate the value of $\Gamma(\omega)$

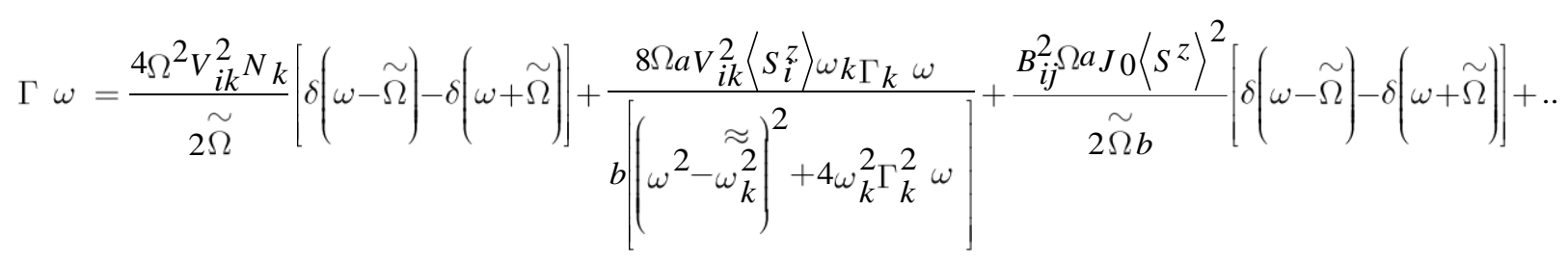

Therefore total $\Gamma(\omega)=\Gamma_{1}(\omega)+\Gamma_{2}(\omega)+\Gamma_{3}(\omega)+\ldots \ldots$

The final Green function is obtained by replacing the $\mathrm{P}(\omega)$ value from equation (6) for the final Green function expressed as;

$$
G \omega=\Omega<S_{i}^{x}>\delta_{i j}\left[\pi\left[\omega^{2}-\widetilde{\Omega}^{2}-P \omega\right]\right]^{-1} .
$$


The various expressions for unmodified frequency and Cochran's mode frequency are given as;

$$
\hat{\Omega}^{2}=\tilde{\Omega}^{2}+2 \Omega \quad \Delta_{4} \omega+\Delta_{5} \omega+\ldots+\Delta_{26} \omega .
$$

In equation (11) we have obtained as

$$
\stackrel{\approx}{\Omega^{2}}=\tilde{\Omega}+2 \Omega \quad \Delta_{1} \omega+\Delta_{2} \omega+\Delta_{3} \omega
$$

and

$$
\widetilde{\Omega^{2}}=4 \Omega^{2}+\frac{i}{\Omega\left\langle S_{i}^{x}\right\rangle}\left\langle\left[F, S_{i}^{y}\right]\right\rangle
$$

Above equation Eqn. is solved by using the mean field approximation, and the value of $\underset{\Omega^{2}}{\sim}$ is

$$
\Omega^{2}=a^{2}+b^{2}-b c
$$

where

$$
a=\left(J+3 J^{\prime}<S_{i}^{z}>^{2}+2 B_{i j}\right)<S_{i}^{z}>,
$$

$b=2 \Omega$ is the modified pseudo spin frequency called

$c=\left(J+3 J^{\prime}<S_{i}^{z}>^{2}+2 B i j\right)<S_{i}^{x}>$,

and

$\widetilde{\Omega}^{2}=\frac{1}{2}\left\{\Omega^{2} \pm 4 \Omega^{2} V_{i k}^{2} N_{k}+B_{i j}^{2} \Omega a J\langle \$ 19\rangle . b j\right.$.

Where $\Omega$ and $\approx$ are pseudo spin frequencies and $\hat{\Omega}$

Cochran mode frequency.

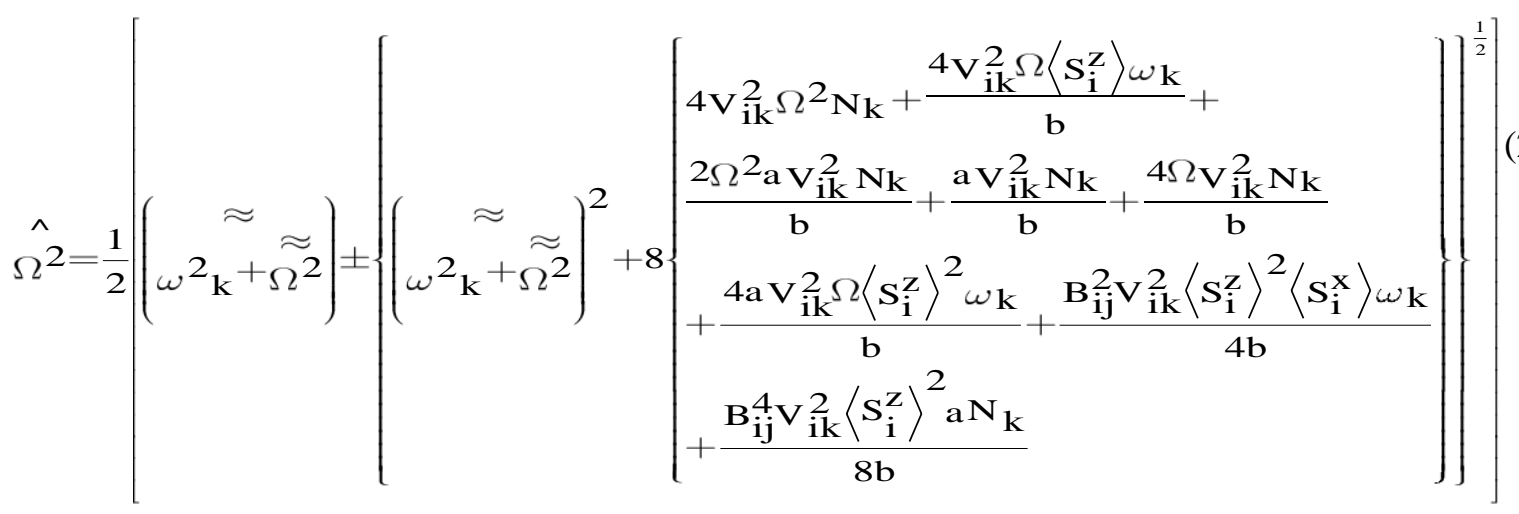


The above expressions contain effects of direct spin-spin interaction, four body interaction and extra spin-lattice interaction. The frequency critically depends upon temperature. If we apply transition condition i.e. $\stackrel{\wedge}{\Omega} \rightarrow 0$ as $\mathrm{T} \rightarrow \mathrm{T}_{\mathrm{c}}$, and in paraelectric phase $\left\langle\mathrm{S}_{\mathrm{i}}^{\mathrm{z}}\right\rangle=0$. The condition gives;

$$
T_{c}=\frac{\Omega}{2 K_{B \tanh }-1\left(\frac{4 \Omega}{J^{\prime}}\right)}
$$

The expressions for electrical permittivity and tangent delta are as follows;

$$
\varepsilon \omega=-8 \pi N \mu^{2} \Omega\left\langle\left\langle S_{i}^{x}\right\rangle\right\rangle\left[\left(\omega^{2}-\Omega^{2}\right)+4 \Omega^{2} \Gamma^{2}\right]^{-1} .
$$

In the $\mathrm{RbH}_{2} \mathrm{PO}_{4}$ ferroelectric crystal, some of the energy is lost in the shape of heat called tangent delta, denoted by $\tan \delta$ expressed as;

$$
\tan \delta=\frac{\varepsilon^{\prime \prime}}{\varepsilon^{\prime}}=-2 \Omega \Gamma \omega\left(\omega^{2-\Omega^{2}}\right)^{-1}
$$

\section{Results and Discussions.}

Now, by fitting the model values of different quantities occurring in expressions from the literature (Ganguli et al, 1980), for $\mathrm{RbH}_{2} \mathrm{PO}_{4}$ crystal as shown in Table (1). We have calculated values of $\left\langle S_{i}^{x}\right\rangle$ and $\left\langle S_{i}^{z}\right\rangle$ for $\mathrm{RbH}_{2} \mathrm{PO}_{4}$ crystal using formulae

$$
\left\langle S_{i}^{x}\right\rangle=\underset{\widetilde{\Omega}}{\frac{2 \Omega}{\sim}} \tanh \frac{\widetilde{\Omega}}{K_{B} T}
$$

and

$$
\left\langle S_{i}^{z}\right\rangle=\frac{\Omega^{2} J_{i j}\left\langle S_{i}^{z}\right\rangle}{\widetilde{\Omega}} \tanh \frac{\widetilde{\Omega}}{K_{B} T} .
$$


Thus we obtain thermal variations of electrical permittivity $(\varepsilon)$, Cochran's mode frequency $\left(\hat{s_{2}}\right)$, and tangent delta $(\tan \delta)$. These quantities are respectively shown in Fig.1, 2, and 3. We observe from Fig.1 at transition temperature $(146 \mathrm{~K})$, the electrical permittivity first increases, from the low-temperature side, being anomalously high at a transition temperature (146K) then decreases above it. From Fig. 2 we infer that Cochran's mode frequency first decreases from the low-temperature side becoming smallest at the transition point (146K) then remains constant above it. We see in Fig.3 that the tangent delta increases from the low-temperature side becoming largest at the transition point and then decreases above it.

Table (1):- Constant values for $\mathrm{RbH}_{2} \mathrm{PO}_{4}$ crystal given by (Ganguli et al, 1980).

\begin{tabular}{|l|l|l|l|l|l|l|l|l|l|l|l|}
\hline $\begin{array}{l}\mathrm{T}_{\mathrm{c}} \\
(\mathrm{K})\end{array}$ & $\begin{array}{l}\mathrm{J}_{1} \mathrm{~cm}^{-} \\
\mathrm{J}^{*} \\
\mathrm{~cm}^{-1}\end{array}$ & $\begin{array}{l}\mathrm{V}_{\mathrm{ik}} \\
\mathrm{cm}^{-1}\end{array}$ & $\begin{array}{l}\Omega \mathrm{cm}^{-} \\
1\end{array}$ & $\begin{array}{l}\omega \mathrm{k} \\
\mathrm{cm}^{-1}\end{array}$ & $\begin{array}{l}\mathrm{C} \\
(\mathrm{K})\end{array}$ & $\begin{array}{l}\mu \\
\text { e.s.u. } \\
\mathrm{cm}^{-1}\end{array}$ & $\begin{array}{l}\mathrm{N} \\
\mathrm{cm}^{-1}\end{array}$ & $\begin{array}{l}B_{i j} \\
\mathrm{~cm}^{-1}\end{array}$ & $\begin{array}{l}N k \\
\mathrm{~cm}^{-3}\end{array}$ & $\begin{array}{l}\mathrm{V}_{\mathrm{ik}}{ }^{\prime} \\
\mathrm{cm}^{-1}\end{array}$ \\
\hline 146.9 & 60 & 20 & 0.252 & 78.40 & 12.5 & 238.90 & $\begin{array}{l}1.8 \\
10^{-18}\end{array}$ & $\begin{array}{l}0.8 \\
10^{21}\end{array}$ & 0.078 & 1.20 & 0.126 \\
\hline
\end{tabular}

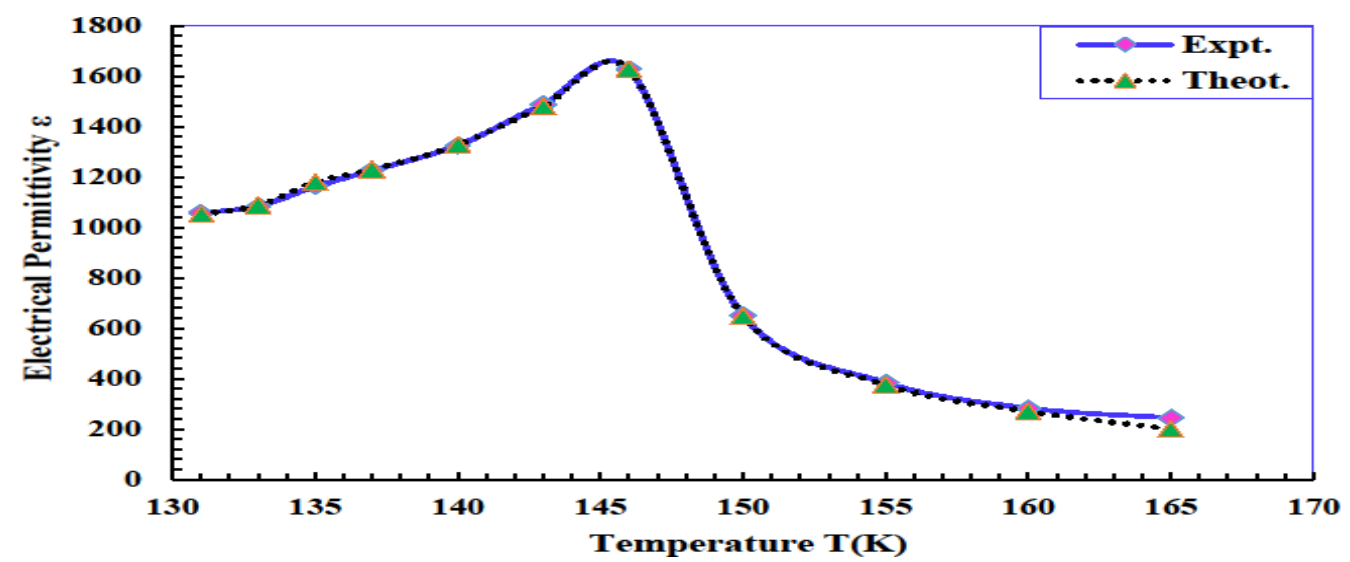

Fig.1-Calculated electrical permittivity $(\varepsilon)$ versus temperature in $\mathrm{RbH}_{2} \mathrm{PO}_{4}$ Crystal; our calculated values denoted by dotted line $\boldsymbol{\Delta}$ correlated with experimental data denoted by a solid line $\downarrow$ of (Peercy et al, 1974).

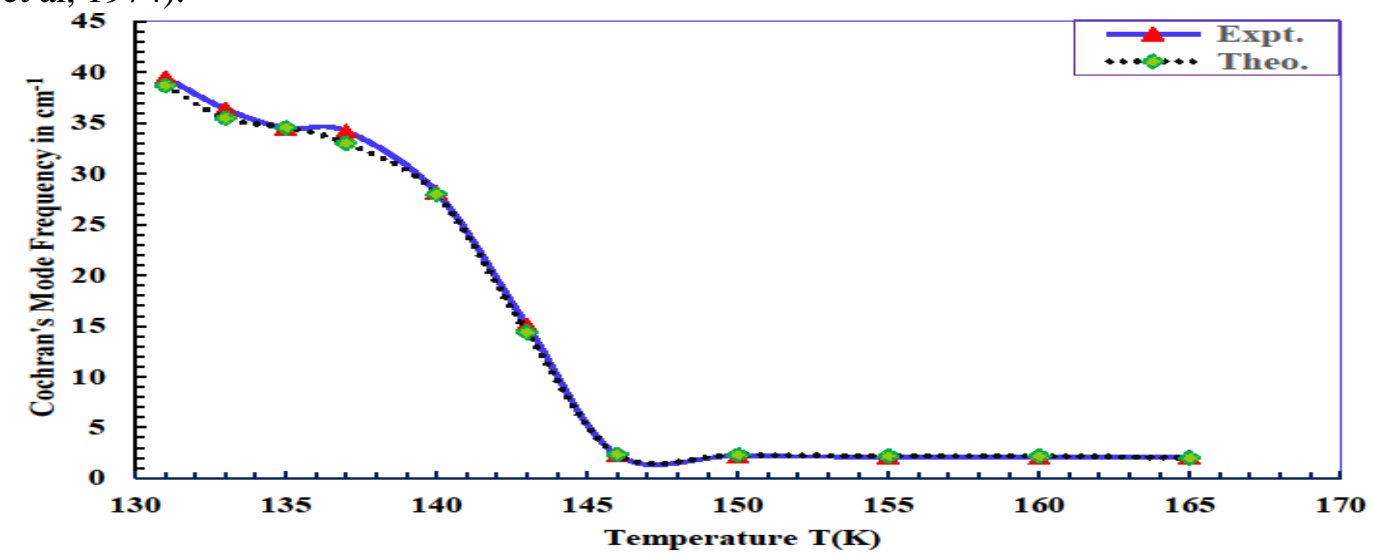

Fig.2-Calculated Cochran's mode frequency $(\hat{\Omega})$ versus temperature $(\mathrm{T})$ in $\mathrm{RbH}_{2} \mathrm{PO}_{4}$ Crystal; our calculated values denoted by dotted line $\boldsymbol{\Delta}$ correlated with experimental data denoted by the solid blue line $\downarrow$ of (Peercy et al, 1974). 


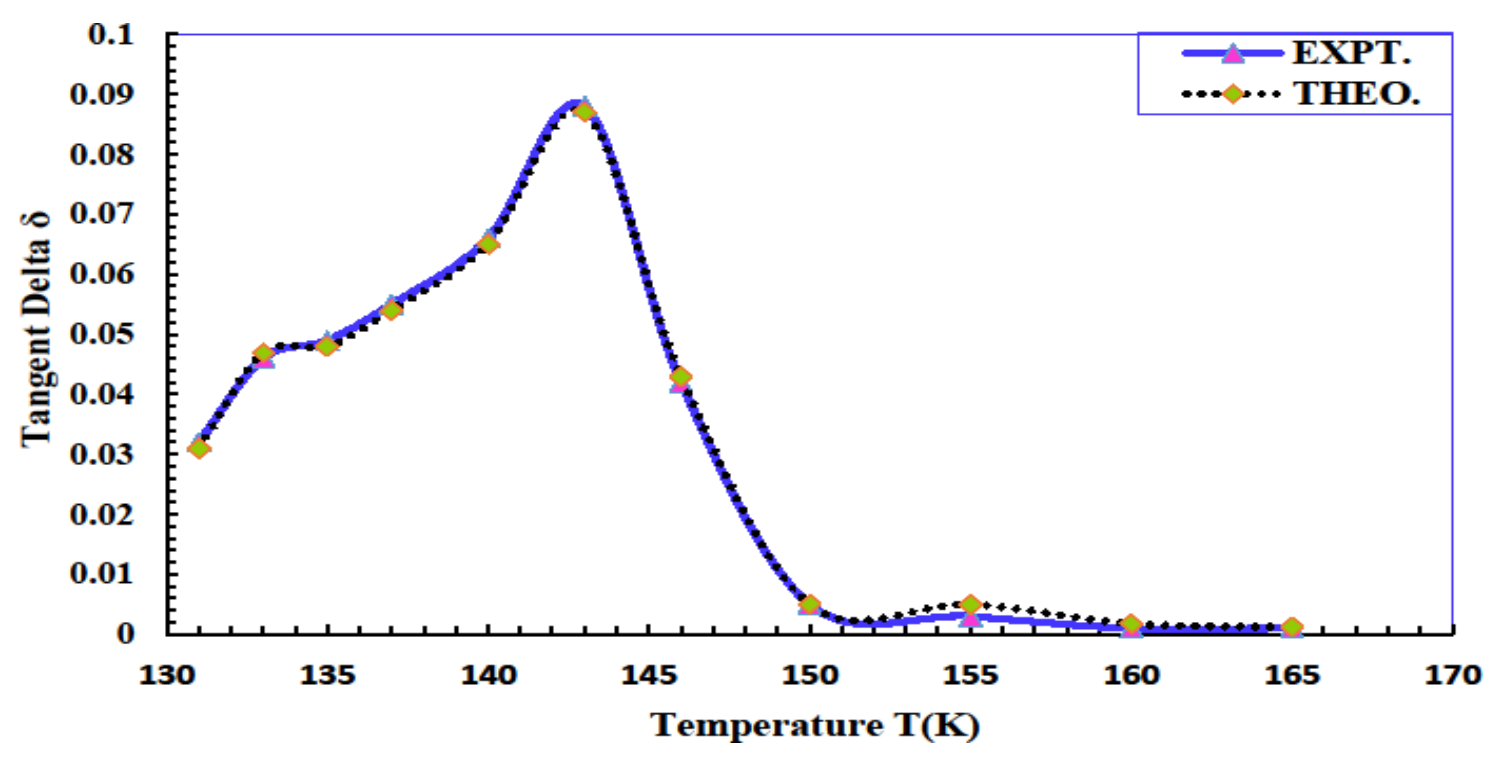

Fig.3-Calculated tangent delta $(\delta)$ versus temperature $(\mathrm{T})$ in $\mathrm{RbH}_{2} \mathrm{PO}_{4}$ crystal; our calculated values denoted by dotted line $\boldsymbol{\Delta}$ correlated with experimental data denoted by the solid blue line $\$$ of (Peercy et al, 1974).

\section{Conclusions}

The work presented in this paper is the extension of the previous author's work (Singh et al, 2021 and Ganguli et al, 1980) with the help of modified Hamiltonian by adding terms for four body interaction, direct spin-spin interaction term, extra spin-lattice interaction term and third and fourth-order anharmonic terms into PLCM model for $\mathrm{RbH}_{2} \mathrm{PO}_{4}$ crystal. We have obtained modified expression for Cochran's mode frequency, electrical permittivity, tangent delta and spontaneous polarization shows that due to other terms considered in the model. We have obtained more rigorous formulae. These formulae show that other interaction terms are important in the ferroelectric $\mathrm{RbH}_{2} \mathrm{PO}_{4}$ crystal which belongs to the family of $\mathrm{KH}_{2} \mathrm{PO}_{4}$ crystals. The present calculation shows that all the interactions considered in the model are important. They contribute to the ferroelectric and dielectric behaviour of

$\mathrm{RbH}_{2} \mathrm{PO}_{4}$ crystal. These terms explain expressions adequately phase transition, Cochran's mode frequency electrical permittivity, tangent delta $\delta$, and Curie temperature $\left(\mathrm{T}_{\mathrm{c}}\right)$, and this type of model will also be applicable for crystals that are isomorphs to $\mathrm{KH}_{2} \mathrm{PO}_{4}$ type crystals.

\section{Acknowledgement}

The authors are grateful to Prof S C Bhatt (EX-HOD), Prof P D Semalty (EX-HOD), Prof O P S Negi (VC-IGOU) Haldwani, Prof Mahavir Singh (EX-VC) Shimla, Prof H C Chandola (HOD-KU Nainital), Dr. Manish Uniyal for their suggestions and encouragement. Kuldeep Kumar is thankful to Mr Pawan Singh, Mr Muzaffar Iqbal Khan, Mr Aditya Joshi, and Mr Mool Chand for encouragement. Kuldeep Kumar is thankful to MOTA, New- Delhi for financial support.

\section{References}

Rbuch-Pouchot, M.T. and Durif, A., (1985). Structure of a new form of rubidium dihydrogenphosphate, RbH2PO4. Acta Crystallographica Section C: Crystal Structure Communications, 41(5), pp.665-667. 
ui, A., Nakai, Y., Mizoguchi, K., Ikeda, S. and Tominaga, Y., (1993). Hydrogen and PO4 modes of RbH2PO4. Journal of the Physical Society of Japan, 62(3), pp.959-964.

Adhav, R.S., (1969). Linear electro-optic effects in tetragonal phosphates and arsenates. JOSA, 59(4), pp.414-418.

Boysen, D.A., Haile, S.M., Liu, H. and Secco, R.A., (2004). Conductivity of potassium and rubidium dihydrogen phosphates at high temperature and pressure. Chemistry of materials, 16(4), pp.693-697.

Blinc, R. and Prelesnik, A., (1960). NMR Study in Rochelle salt. The Journal of Chemical Physics, 32(2), pp.387-388.

Cochran, W., (1960). Crystal stability and the theory of ferroelectricity. Advances in Physics, 9(36), pp.387-423.

Courtens, E. and Vogt, H., (1985). Raman study of rubidium-ammonium dihydrogen phosphate glasses. Journal de chimie physique, 82, pp.317-327.

Dalterio, R.A. and Owens, F.J., (1988). A Raman scattering study of high-temperature phase transitions in potassium and rubidium dihydrogen phosphate. Journal of Physics C: Solid StatePhysics, 21(36), p.6177.

Ganguli, S., Nath, D. and Chaudhuri, B.K., (1980). Green's-function theory of phase transitions in hydrogen-bonded ferroelectric crystals with pseudo-spin-lattice coupled mode model. Physical Review B, 21(7), p.2937.

Khan, M.I., Singh, P. and Upadhyay, T.C., (2021). Study of ferroelectric phase transition in H-bonded triglycine selenate (TGSe) crystal. Materials Today: Proceedings.

Khan, M.I. and Upadhyay, T.C., (2020). Phase transition thermal dependence of ferroelectric and dielectric properties in H-bonded \$ \$hbox $\{\mathrm{PbHPO}\}_{-}\{4\} \$ \$$ PbHPO 4 (LHP) crystal. Applied PhysicsA, 126(11), pp.1-10.

Mattauch, S., Heger, G. and Michel, K.H., (2004). High resolution neutron and X-ray diffraction studies as a function of temperature and electric field of the ferroelectric phase transition of RDP.

Crystal Research and Technology: Journal of Experimental and Industrial Crystallography, 39(12), pp.1027- 1054.

Peercy, P.S., (1974). Evaluation of the coupled proton-optic-mode model for K H 2 P O 4 and Rb H 2 P O 4. Physical Review B, 9(11), p.4868.

Semwal, B.S. and Sharma, P.K., (1974). Dielectric susceptibility and infrared absorption in an anharmonic crystal. Progress of Theoretical Physics, 51(3), pp.639-655.

Upadhyay, T.C. and Semwal, B.S., (2002). Temperature dependence of dielectric tangent loss in KDP and DKDP crystals at microwave frequencies.

Zubarev, D., (1960). Double-time Green functions in statistical physics. Soviet Physics Uspekhi, 3(3), p.320. 